\title{
Context Switching Algorithm for Selective Multibiometric Fusion
}

\author{
Mayank Vatsa ${ }^{1}$, Richa Singh $^{1}$, and Afzel Noore ${ }^{2}$ \\ 1 IIIT Delhi, India \\ mayank@iiitd.ac.in, rsingh@iiitd.ac.in \\ 2 West Virginia University, USA \\ afzel.noore@mail. wvu.edu
}

\begin{abstract}
This paper presents a multimodal biometric fusion algorithm that supports biometric image quality and case-based context switching approach for selecting appropriate constituent unimodal traits and fusion algorithms. Depending on the quality of input samples, the proposed algorithm intelligently selects appropriate fusion algorithm for optimal performance. Experiments and correlation analysis on a multimodal database of 320 subjects show that the context switching algorithm improves the verification performance both in terms of accuracy and time.
\end{abstract}

\section{Introduction}

A biometric system operates by acquiring biometric data from an individual, extracting a feature set from the acquired data, and comparing this feature set against known templates stored in the database. Further, the use of multiple biometric samples or evidences to verify the identity of an individual is often referred to as multibiometrics. Multimodal systems have several advantages over unimodal biometric systems such as resiliency to noise and malfunction, universality, and improved accuracy. Researchers have also shown that fusion of multiple biometric evidences, in general, enhances the recognition performance. Over the years, several fusion algorithms have been proposed and a comprehensive review of existing algorithms are presented in [1]. Currently, many law enforcement agencies use face, fingerprint and iris to authenticate the identity of an individual. Existing commercial systems such as HIIDE, capture these modalities and process them individually. However, the system does not fuse individual match scores or decisions to further obtain reliable and improved performance. Very limited research exists in making a paradigm shift toward an approach that can dynamically perform selective fusion and intelligently use image quality metric in the fusion framework.

This paper focuses on the need to enhance the capability to recognize individuals operating in uncontrolled environment that is common in many real world situations. In this research we develop an adaptive biometric fusion algorithm to efficiently match individuals using multiple modalities even when the biometric samples are non-optimal. Specifically, we design a context switching tool that

S. Chaudhury et al. (Eds.): PReMI 2009, LNCS 5909, pp. 452-457, 2009.

(C) Springer-Verlag Berlin Heidelberg 2009 
can dynamically select the most appropriate constituent unimodal classifier or the most appropriate fusion algorithm for the given set of probe images using image quality scores. The concept of context switching can be stated as follows:

"When dealing with gallery-probe pairs of good quality, any efficient unimodal classifier can verify the identity without the need for fusion. When the quality of image falls in the range of good to average, biometric classifiers yield some conflicting results. For such cases, simple fusion rules such as sum rule with min-max normalization [1] can successfully fuse the match scores and yield correct results with very less time complexity. When dealing with non-optimal gallery probe samples due to poor image quality or availability of partial images, complex fusion rules using support vector machines are required to perform fusion. The proposed context switching tool reconciles constituent biometric classifiers (e.g. face, fingerprint, and iris recognition algorithms) with a set of fusion algorithms that contains both simple and complex schemes to optimize both verification accuracy and computational time."

On the multimodal biometric database of 320 subjects, the proposed context switching fusion algorithm improves the verification accuracy and reduces the computational cost of the system compare to existing fusion algorithms.

\section{Proposed Context Switching Algorithm}

Fig. 1 illustrates the steps involved in the proposed dynamic context switching algorithm. For a biometric system with two classes (genuine, impostor) and three modalities, the algorithm uses image quality scores and three classifiers (e.g. decision tree or support vector machine (SVM)) for context switching. Classifier1 is used to choose between the unimodal and multimodal approach based on the input evidences. If the quality of probe image is above a non-linear threshold, then unimodal approach is selected otherwise multimodal approach is selected. Next, if the unimodal approach is selected, then Classifier-2 is used to select one of the three unimodal options: (1) only face, (2) only fingerprint, and (3) only iris. If Classifier- 1 selects the multimodal approach, then Classifier- 3 is used to select the optimal fusion rule for a given probe case. Classifier-3 selects a complex fusion algorithm only when there is uncertainty or imperfection in the image quality scores otherwise it selects a simple fusion algorithm for combining information obtained from multimodal biometric images.

\subsection{Design of Algorithm}

In the context switching algorithm, three SVMs are used as the three classifiers to reconcile unimodal algorithms and fusion algorithms. Input to the first SVM, denoted as $S V M_{1}$, is used to select unimodal algorithms (face, fingerprint, and iris) or fusion rules. If unimodal algorithms are selected then the second SVM, denoted as $S V M_{2}$, is used to choose among face, fingerprint, and iris. If the option pertaining to fusion rules is selected then the third SVM, denoted as $S V M_{3}$, is used to select the optimal fusion algorithm among a collection of 


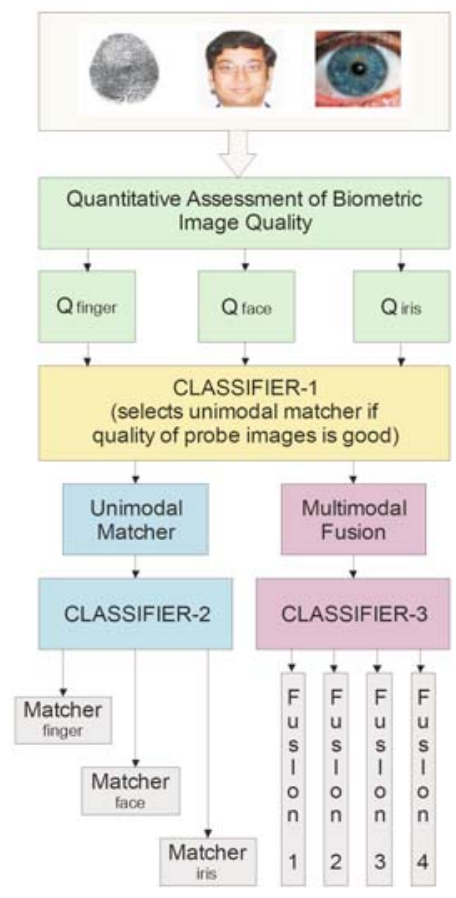

Fig. 1. Illustrating the concept of the proposed context switching algorithm

fusion rules. The context switching algorithm is divided into two stages: training SVMs for reconciliation and dynamic selection for every query instance.

Training SVMs for Reconciliation: Three SVMs are independently trained using the labeled training database. The training procedure is explained below.

1. $S V M_{1}$ is two-class classifier that is trained using the labeled training data $\left\{x_{1 i}, y_{1 i}\right\}$. Here, $x_{1 i}$ is the quality vector belonging to the $i^{\text {th }}$ training galleryprobe pair. $y_{1 i} \in(+1,-1)$ is the respective label such that +1 is assigned when the gallery-probe pair is of high quality and can be correctly matched using unimodal algorithm and -1 is assigned to the data that requires fusion.

2. $S V M_{2}$ is a multiclass classifier that is trained using the labeled training data $\left\{x_{2 i}, y_{2 i}\right\}$ where, $x_{2 i}$ is the quality vector belonging to the $i^{\text {th }}$ training gallery-probe pair and $y_{2 i}$ is the multiclass soft label. In the soft labeling, range of $[-1,-0.66]$ belongs to gallery-probe pair that can be matched using face recognition, range of $[-0.66,0.33]$ belongs to gallery-probe pair that can be fused using fingerprint recognition, and $[0.33,1]$ is assigned to the data that requires matching with iris recognition.

3. $S V M_{3}$ is also a multiclass classifier that is trained using the labeled training data $\left\{x_{3 i}, y_{3 i}\right\}$. Here, $x_{3 i}$ is the $i^{\text {th }}$ training data vector that contains quality scores, match scores and verification accuracy priors pertaining to the three 
unimodal recognition algorithms, and $y_{3 i}$ is the soft label such that $S V M_{3}$ classifies the constituent collection of fusion rules.

Dynamic Context Switching at Probe level: For probe verification, the trained SVMs are used to dynamically select the most appropriate algorithm depending on the quality scores.

1. The quality scores pertaining to both the gallery-probe images are provided as input to the trained SVMs. $S V M_{1}$ classifier selects between unimodal algorithms and fusion rules.

2. To improve the performance, the classification result of $S V M_{1}, S V M_{2}$ or $S V M_{3}$ are used to select one of the four options: (1) only face, (2) only fingerprint, (3) only iris, and (4) optimal fusion rule of the given probe images. The selected algorithm is then used for final decision-making.

\subsection{Details of Implementation}

In implementing the algorithm, we use existing algorithms for computing quality scores for face, fingerprint and iris images, and for extracting biometric features. Specifically, for computing face image quality score we use quality assessment algorithm describe in 2, RDWT based algorithm [3] is used for computing fingerprint quality score and Dempster Shafer theory based algorithm [4] for iris image quality assessment. Further, neural network architecture based Gabor transformation [5] is used to extract facial features, state-of-the-art commercial fingerprint and iris feature extraction and matching tools are used for fingerprint and iris recognition [6]. Furthermore, we use sum rule with min-max normalization [1] and likelihood ration based SVM fusion (referred as LR-SVM) [7] as two constituent fusion rules. Note that, in this paper, we use two score level fusion rules, however it can include other levels of fusion such as image or feature fusion. Finally, to train SVMs, we use radial basis kernel with kernel parameter of 4 (it our experiments we observe that it yields the best accuracy) and to compute soft labels, we use standard density estimation approach 8 .

\section{Experimental Evaluation and Discussion}

Evaluation is performed on West Virginia University (WVU) multimodal database. This database contains face, fingerprint, and iris images from 320 subjects. The database is divided into two partitions: train and test. Training database is composed of face, fingerprint, and iris images pertaining to 128 subjects ( $40 \%$ of total population) and rest of the images pertaining to 192 subjects are used as test (gallery-probe) data. We also perform 20 times cross validation and receiver operating characteristics (ROC) curves are generated across these trials. Verification accuracies are reported at $0.01 \%$ false accept rate (FAR). Fig. 2 shows the ROC curves along with verification performance (accuracy and time) of the proposed algorithm and comparison with constituent unimodal and match score fusion algorithms. The key analysis of the results are delineated as: 


\begin{tabular}{|l|l|l|}
\hline Algorithm & $\begin{array}{l}\text { Verification } \\
\text { Accuracy } \\
\text { At } 0.01 \% \text { FAR }\end{array}$ & $\begin{array}{l}\text { Time } \\
(\mathrm{sec} .)\end{array}$ \\
\hline Face & 84.9 & 2.2 \\
\hline Fingerprint & 76.1 & 1.0 \\
\hline Iris & 92.0 & 0.4 \\
\hline $\begin{array}{l}\text { Sum Rule + Min-Max } \\
\text { Normalization }\end{array}$ & 94.4 & 4.1 \\
\hline LR-SVM Fusion & 97.1 & 5.4 \\
\hline Proposed & 97.2 & 3.3 \\
\hline
\end{tabular}

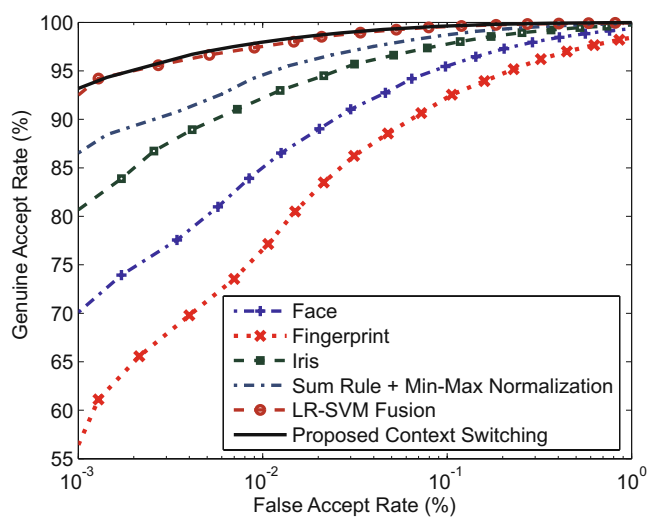

Fig. 2. Performance of the proposed context switching algorithm and comparison with unimodal and existing match score fusion algorithms

- We evaluate the correlation among biometric sources by estimating the Pearsons correlation coefficient and Spearmans rank correlation measure to model dependencies in match scores. We observe that fusing negatively correlated information sources results in pronounced improvement in matching performance which is in accordance to results of other researchers [9].

- The results show that among the unimodal traits, iris is among the best and fingerprint provides an accuracy of $76.1 \%$. Lower performance of fingerprint is due to large variation in image quality and sensor noise.

- Both the fusion rules improve the verification accuracy by 2.4-5.1\%. More importantly, correlation analysis between two fusion rules suggests that there are cases when sum rule yields better accuracy compare to LR-SVM fusion and vice-versa.

- The correlation analysis of unimodal and multimodal scores also supports the underlying concept of context switching. Experimental results show that the proposed context switching algorithm not only improves the verification accuracy, though slightly on WVU-multimodal database, but also decreases the average verification time. On 2.4 Duo Core processor with 2GB RAM under Matlab environment, the proposed algorithm require 3.3 seconds whereas existing fusion rules require 4.1-5.4 seconds.

- By design, another important aspect of the proposed context switching algorithm is that it can be easily modified to include other biometric modalities and fusion rules.

\section{Conclusion}

The paradigm of information fusion entails in processing evidence presented by multiple sources to enhance the recognition performance of biometric systems. Although extensive research has been done, there is a need to enhance the 
recognition capability when operating in uncontrolled environment. This paper presents a context switching algorithm that can fill the gap in the current stateof-art. The proposed algorithm analyzes the input biometric samples obtained from diverse, disparate sensors and characterizes the samples based on the quality and amount of information present. It further performs context switching by adaptively assessing if a unimodal biometric classifier can reliably identify an individual with high accuracy or it is required to choose the most appropriate multimodal biometric fusion algorithm based on the degree of uncertainty, incompleteness, and distortion present in the biometric samples. The proposed algorithm optimizes the underlying algorithmic and computational challenges in the decision making process such that the performance with respect to both accuracy and response time guarantees the success in real world operational scenario.

\section{References}

1. Ross, A., Nandakumar, K., Jain, A.: Handbook of multibiometrics. Springer, Heidelberg (2006)

2. Vatsa, M., Singh, R., Noore, A.: SVM based adaptive biometric image enhancement using quality assessment. In: Speech, Audio, Image and Biomedical Signal Processing using Neural Networks, vol. 83, pp. 351-371. Springer, Heidelberg (2008)

3. Vatsa, M., Singh, R., Noore, A., Houck, M.: Quality-augmented fusion of level2 and level-3 fingerprint information using DSm theory. International Journal of Approximate Reasoning 50(1), 51-61 (2009)

4. Kalka, N.D., Zuo, J., Dorairaj, V., Schmid, N.A., Cukic, B.: Image quality assessment for iris biometric. In: Proceedings of SPIE Conference on Biometric Technology for Human Identification III, vol. 6202, pp. 61020D-1-62020D-11 (2006)

5. Singh, R., Vatsa, M., Noore, A.: Face recognition with disguise and single gallery images. Image and Vision Computing 27(3), 245-257 (2009)

6. http://www . neurotechnology.com

7. Vatsa, M., Singh, R., Ross, A., Noore, A.: Likelihood ratio in a SVM framework: fusing linear and non-linear classifiers. In: Proceedings of IEEE Computer Society Workshop on Biometrics at Computer Vision and Pattern Recognition Conference, pp. 1-6 (2008)

8. Tao, Q., Wu, G., Wang, F., Wang, J.: Posterior probability support vector machines for unbalanced data. IEEE Transaction on Neural Network 16(6), 1561-1573 (2005)

9. Kuncheva, L.I., Whitaker, C.J., Shipp, C.A., Duin, R.P.W.: Is independence good for combining classifiers? In: Proceedings of International Conference on Pattern Recognition, vol. 2, pp. 168-171 (2000) 